\title{
A NOTE ON GLOBAL SOLVABILITY OF VECTOR FIELDS
}

\author{
JORGE HOUNIE ${ }^{1}$
}

\begin{abstract}
We consider global solvability of complex vector fields on noncompact manifolds. The case of real vector fields had been considered by Malgrange, and Hörmander studied the complex case, assuming that the real and imaginary parts are everywhere linearly independent.
\end{abstract}

1. We consider a smooth complex vector field $L=X+i Y$ without zeros on a smooth, paracompact, noncompact manifold $M$. The real vector fields $X$ and $Y$ do not vanish simultaneously. The pair of vectors $X$ and $Y$ defines an everywheredefined group of local diffeomorphisms in $M[8, \mathrm{p} .175]$. The equivalence classes defined by this group (two points, $a$ and $b$, are related if there is a local diffeomorphism in the group that takes $a$ into $b$ ) will be called the orbits of $L$ in $M$. The orbits are connected submanifolds of $M$ with a natural differentiable structure. If $U \subseteq M$ is open, the orbit of $L$ in $U$ through the point $p$ is contained in, but not necessarily equal to, the intersection of $U$ with the orbit of $L$ in $M$ through $p$.

We shall deal with the global solvability of the equation $(L+a) u=f$ with $u$ and $f$ smooth, and $a$ smooth and fixed. If $L+a$ is surjective in $C^{\infty}(M)$, it follows that $L$ should verify condition (P) in $M[5]$. Condition (P) implies [3] that the orbits of $L$ have dimension one or two. We shall assume that:

(1.1) No orbit is relatively compact in $M$.

This condition is not necessary for the solvability of $L+a$, but it implies the sufficient conditions for semiglobal solvability given in [4].

Assume furthermore that:

(1.2) For each compact set $K$ of $M$ there exists a compact set $K^{\prime}$ such that if $B$ is an orbit of $L$ in $M \backslash K$ which is relatively compact in $M$, then $B \subseteq K^{\prime}$.

If (1.1) holds, (1.2) can be reformulated for one-dimensional orbits as follows:

$(1.2)^{\prime}$ For each compact set $K$ of $M$ there exists a compact set $K^{\prime} \subseteq M$ such that if $\gamma$ is a one-dimensional orbit of $L$ in $M \backslash K$ with endpoints in $K$, it follows that $\gamma \subseteq K^{\prime}$.

THEOREM 1.1. If $L$ verifies $(\mathrm{P}),(1.1)$ and (1.2), then

$$
(L+a) C^{\infty}(M)=C^{\infty}(M) \quad \forall a \in C^{\infty}(M) .
$$

In the special cases where (i) $X$ and $Y$ are linearly independent everywhere, or (ii) $M$ is an open subset of the plane, Theorem 1.1 follows from $[2, \S 7.1$, and 6].

When $\operatorname{dim} M=2$ it is possible to make these results more precise.

THEOREM 1.2. Assume that $L$ satisfies (1.1) and $\operatorname{dim} M=2$. Then $(L+a) C^{\infty}(M)=C^{\infty}(M)$ if and only if $L$ satisfies $(\mathrm{P})$ and $(1.2)^{\prime}$.

Received by the editors April 18, 1984.

1980 Mathematics Subject Classification. Primary 33A05, 35F05.

${ }^{1}$ The author was partially supported by $\mathrm{CNP}_{\mathrm{q}}$ (Brazil). 
2. If $E_{1}$ and $E_{2}$ are smooth vector bundles over $M$, denote by $\Gamma^{k}\left(E_{i}\right)$ the space of $C^{k}$ cross-sections of $E_{i}$ with its natural topology and by $\varepsilon^{\prime k}\left(E_{i}\right)$ its dual $i=1,2$. If $K$ is a compact subset of $M, \Gamma^{k}\left(K, E_{1}\right)$ denotes the quotient of $\Gamma^{k}\left(E_{i}\right)$ by the subspace consisting of elements vanishing of order $k$ on $K$. The dual of $\Gamma^{k}\left(K, E_{i}\right)$ is denoted by $\varepsilon^{\prime k}\left(K, E_{i}\right)$; it is naturally injected into $\varepsilon^{\prime k}\left(E_{i}\right)$.

We shall use the following version of a theorem of Malgrange [7].

THEOREM 2.1. The following conditions are equivalent for a differential operator $P: \Gamma^{\infty}\left(E_{1}\right) \rightarrow \Gamma^{\infty}\left(E_{2}\right)$ :

(i) $P\left(\Gamma^{\infty}\left(E_{1}\right)\right)=\Gamma^{\infty}\left(E_{2}\right)$,

(ii) $P\left(\Gamma^{\infty}\left(K, E_{1}\right)\right)=\Gamma^{\infty}\left(K, E_{2}\right)$ for all compact subsets $K$ of $M$.

\section{Furthermore:}

(2.1) For each compact subset $K$ of $M$ and any positive integer $k$, there is a compact set $K^{\prime} \subset M$ such that if $v \in \varepsilon^{\prime \infty}\left(E_{2}\right)$, then ${ }^{t} P(v) \in \varepsilon^{\prime k}\left(K, E_{1}\right)$ implies $v \in \varepsilon^{\prime}\left(K, E_{2}\right)$.

Here ${ }^{t} P$ is the dual operator of $P$.

In the following corollary, $\operatorname{Fr} V$ denotes the boundary of an open set $V$.

COROLLARY 2.2. Let $M$ be a smooth manifold of dimension $n$. Then for each compact subset $K$ of $M$, there is a compact subset $\hat{K}$ of $M$ such that if $V \subseteq M$ is open and relatively compact, $\operatorname{Fr} V \subseteq K$ implies $V \subseteq \hat{K}$.

ProOF. We may assume $M$ is not compact. Thus, the $n$th de Rham cohomology space $H^{n}(M)=0$. This means that $d_{n}\left(\bigwedge^{n-1} C^{\infty}(M)\right)=\bigwedge^{n} C^{\infty}(M)$. Assuming that $M$ is orientable, we may identify ${ }^{t} d_{n}$ with $-d_{1}: \varepsilon^{\prime}(M) \rightarrow \bigwedge^{1} \varepsilon^{\prime}(M)$ using the pairings

$$
\bigwedge^{k} C_{k}^{\infty}(M) \times \bigwedge^{n-k} C^{\infty}(M) \ni(\alpha, \beta) \mapsto \int \alpha \wedge \beta, \quad k=0,1 .
$$

Now we can apply (2.1) with $k=1$ to the characteristic function $v$ of $V$ to obtain the result in this case. When $M$ is not orientable we apply the previous reasoning to the orientable double cover of $M$. Q.E.D.

In the analysis of condition (2.1), the proposition below will be useful. It is a standard consequence of the fact that first-order operators that verify $(P)$ possess uniqueness in the Cauchy problem with respect to noncharacteristic surfaces in the class of distributions $[\mathbf{1}]$.

Proposition 2.3. Assume that the vector field $L$ verifies (P) in $M$. If $u \in$ $D^{\prime}(M)$ satisfies $(L+a) u=0$, then $\operatorname{supp} u$ is a union of orbits of $L$.

ProOF. We need only show that if $\gamma:[a, b] \rightarrow M$ is an integral curve of $\pm X$ or $\pm Y$ which does not reduce to a point and $u$ vanishes in a neighborhood of $\gamma(a)$, then $u$ vanishes in a neighborhood of $\gamma(b)$. We may consider coordinates $x_{1}, \ldots, x_{n}$ in a neighborhood $U$ of $\gamma$ so that

$$
U=\left\{a-\delta<x_{1}<b+\delta, \sum_{j=2}^{n} x_{j}^{2}<1\right\}, \quad \gamma(t)=\{t, 0, \ldots, 0\}, \quad u \equiv 0,
$$


in $U$ for $x_{1}<a+\delta$ and, say, $\partial / \partial x^{1}=X$. Consider a one-parameter family of smooth surfaces in $U, \varphi(x, \varepsilon)=0, x \in U, 0<\varepsilon<1$, such that the subsets $V_{\varepsilon}=\left\{\varphi_{\varepsilon} \leq 0\right\}$ verify

(i) $V_{\varepsilon} \subset V_{\varepsilon^{\prime}}$, if $\varepsilon<\varepsilon^{\prime}$,

(ii) $\bigcup_{0<\varepsilon<1} V_{\varepsilon}=U$,

(iii) $V_{1 / 2} \subseteq\left\{X_{1}<a\right\}$,

(iv) $\overline{V_{\varepsilon}} \cap\left\{X_{1} \geq a\right\}$ is a compact subset of $U, 0<\varepsilon<1$,

(v) $\varphi_{\varepsilon}=0$ is noncharacteristic with respect to $\partial / \partial x^{1}, 0<\varepsilon<1$.

Such a family is easily constructed. Let $\varepsilon_{0}=\sup \varepsilon$, where the supremum is taken over those $\varepsilon$ 's for which $V_{\varepsilon} \cap \operatorname{supp} u=\varnothing$. If $\varepsilon_{0}<1$ there is a point $p$ in supp $u \cap U$ such that $\varphi\left(p, \varepsilon_{0}\right)=0$ and $u$ vanishes on $\varphi\left(p, \varepsilon_{0}\right) \leq 0$. Since $\varphi\left(x, \varepsilon_{0}\right)=0$ is noncharacteristic for $L$ and $L u=0, u$ vanishes in a neighborhood of $p$, a contradiction. Therefore $\varepsilon_{0}=1$ and $u \equiv 0$ in $U$. Q.E.D.

Assume that $L$ satisfies (1.1) and (P), and let $(p, \xi)$ be a characteristic point of $L$ in $T^{*}(M) \backslash 0$ over a compact set $K$. Since the orbit of $p$ is not contained in $K$, we may find an integral curve $\gamma$ of $\alpha X-\beta Y=\operatorname{Re}[(\alpha+i \beta) L]$ for certain $\alpha, \beta$ in $C^{\infty}(M)$ so that $\gamma$ joins $p$ to a point outside $K$. The curve $\gamma$ is the projection of a semibicharacteristic that joins $p$ to a point over the complement of $K$. The results of [4] imply that $(L+a) C^{\infty}(K)=C^{\infty}(K)$ for all compact subsets of $M$. Assume furthermore that $L$ satisfies (1.2), and consider the transpose ${ }^{t} P=-(L+b)$ of $P=L+a$. If $K$ is a compact subset of $M$ and $K^{\prime} \supset K$, the corresponding subset in (1.2), we claim that, for $u \in \varepsilon^{\prime}(M),{ }^{t} P u \in \varepsilon^{\prime}(K)$ implies $u \in \varepsilon^{\prime}\left(K^{\prime}\right)$. Indeed, let $p \in \operatorname{supp} u \backslash K$ and consider the orbit $B$ of $L$ in $M \backslash K$ through the point $p$. Since ${ }^{t} P u=0$ in $M \backslash K$, it follows from Proposition 2.3 that $B \subseteq \operatorname{supp} u \backslash K$. Since $B$ is relatively compact in $M, B \subseteq K^{\prime}$ and, in particular, $p \in K^{\prime}$. Now, we can apply Theorem 2.1 to $P$ to conclude that $P$ is surjective in $C^{\infty}(M)$. This proves Theorem 1.1.

To prove Theorem 2.2 we need two lemmas. We leave to the reader the proof of the first one.

LEMMA 2.4. Assume that $\operatorname{dim} M=2$ and let $B$ be a two-dimensional orbit of $L$. Then $B$ is open and $\operatorname{Fr} B$ is a union of one-dimensional orbits.

LEMMA 2.5. If $\operatorname{dim} M=2$ and (1.1) holds, (1.2) and (1.2)' are equivalent.

ProOF. We need only show that (1.2)' implies (1.2). Let $K$ be a compact subset of $M, K^{\prime}$ the corresponding subset of property $(1.2)^{\prime}$, and $K_{1}=\widehat{K}^{\prime}$ the subset corresponding to $K^{\prime}$ in Corollary 2.2. If $\gamma$ is a one-dimensional orbit of $L$ in $M \backslash K$ which is relatively compact in $M$, then $\gamma$ has two endpoints in $K$ (otherwise $\gamma$ would possess an $\alpha$-limit point or an $\omega$-limit point, and the orbit through the limit point would be relatively compact in $M)$. Hence $\gamma \subseteq K^{\prime}$. Let $B$ be a two-dimensional orbit in $M \backslash K$ with compact closure in $M$. In view of Corollary 2.2 it is enough to prove that $\operatorname{Fr} B \subseteq K^{\prime}$. Since $\operatorname{Fr} B$ is compact, it follows that if $p \in \operatorname{Fr} B \backslash K, p$ is contained in a one-dimensional orbit $\gamma$ in $M \backslash K$ with two endpoints in $K$. Again $(1.2)^{\prime}$ implies that $\gamma \subseteq K^{\prime}$. Q.E.D.

The "if" part of Theorem 1.2 follows from Theorem 1.1 and Lemma 2.5. To complete the proof consider a subset $K$ of $M$, and let $K^{\prime}$ be the subset in (2.1) with $k=1$. If $\gamma:[a, b] \rightarrow M \backslash K$ is a one-dimensional orbit in $M \backslash K$ with endpoints 
in $K$, we may take coordinates in a neighborhood $U$ of $\gamma([a, b])$ so that

$$
\begin{gathered}
U=\left\{a-\delta<x_{1}<b+\delta,\left|x_{2}\right|<1\right\}, \quad \gamma(t)=(t, 0), \quad a \leq t \leq b, \\
{ }^{t} P=q(x)\left(\frac{\partial}{\partial x^{1}}+i \beta(x) \frac{\partial}{\partial x^{2}}+\gamma(x)\right), \quad q \neq 0,
\end{gathered}
$$

and $\beta\left(x_{1}, 0\right)=0$ if $a \leq x_{1} \leq b$. Set $u=\varphi \psi \otimes \delta \in \varepsilon^{\prime}(U)$, where

$$
\varphi\left(x_{1}\right)=\exp \left\{\int_{0}^{x_{1}}\left(i \frac{\partial \beta}{\partial x^{\prime}}(s, 0)-\gamma(s, 0)\right) d s\right\},
$$

$\psi\left(x_{1}\right)$ is the characteristic function of the interval $[a, b]$ and $\sigma\left(x_{2}\right)$ is the Dirac measure. Then ${ }^{t} P u \in \varepsilon^{\prime 1}(U) \subseteq \varepsilon^{\prime 1}(M)$ and $\operatorname{supp}\left({ }^{t} P u\right)$ consists of the two endpoints $\gamma(a)$ and $\gamma(b)$ contained in $K$. Thus, $\operatorname{supp}(u)=\gamma([a, b]) \subseteq K^{\prime}$. Q.E.D.

\section{REFERENCES}

1. F. Cardoso and J. Hounie, First onder linear PDE's and uniqueness in the Cauchy problem, J. Differential Equations 33 (1979).

2. J. Duistermaat and L. Hörmander, Fourier integral operators. II, Acta Math. 128 (1972).

3. J. Hounie, Globally hypoelliptic vector fields on compact surfaces, Comm. Partial Differential Equations 7 (1982).

4. L. Hörmander, Propagation of singularities and semi-global existence theorems for (pseudo-) differential operators of principal type, Ann. of Math. (2) 108 (1978).

5. __ Pseudo-differential operators of principal type, Singularities in Boundary Value Problems, NATO Adv. Study Inst. Ser., Nijhoff, The Hague, 1981.

6. D. Kim, Ph.D. Thesis, Rutgers Univ., 1981.

7. B. Malgrange, Existence et approximation des solutions des équations aux dérivées partielles et des équations de convolution, Ann. Inst. Fourier (Grenoble) 6 (1955-56).

8. H. Sussmann, Orbits of families of vector fields and integrability of distributions, Trans. Amer. Math. Soc. 180 (1973).

Departmento de Matematica, Universidade Federal de Pernambuco, 50000 RECIFE, PE, BRAZIL 